\title{
Phytochemical and antioxidant characterization of Hypericum perforatum alcoholic extracts
}

\author{
Bruno A. Silva ${ }^{\text {a }}$, Federico Ferreres ${ }^{\text {b, João O. Malva }}{ }^{\text {, }}$, Alberto C.P. Dias ${ }^{\text {a,* }}$

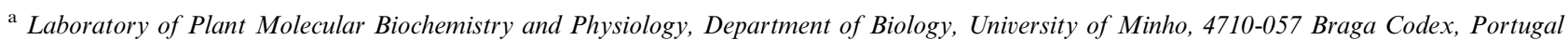 \\ ${ }^{\mathrm{b}}$ Research Group on Quality, Safety and Bioactivity of Plant Foods, CEBAS (CSIC), P.O. Box 164, E-30100 Espinardo (Murcia), Spain \\ ${ }^{\mathrm{c}}$ Institute of Biochemistry and Center for Neuroscience and Cell Biology of Coimbra, Faculty of Medicine, University of Coimbra, \\ 3004-504 Coimbra, Portugal
}

Received 19 January 2004; received in revised form 11 March 2004; accepted 11 March 2004

\begin{abstract}
The antioxidant potentials of a total ethanolic extract of Hypericum perforatum (TE) and fractions were evaluated and correlated with their phenolic contents. The extracts were fully characterised by HPLC-DAD-MS-MS. Kaempferol 3-rutinoside and rutinacetyl were identified for the first time in TE extracts. The free radical-scavenging properties of $\mathrm{TE}\left(\mathrm{EC}_{50}=21 \mu \mathrm{gdwb} / \mathrm{ml}\right)$ and fractions were studied using DPPH. Fractions containing flavonoids and/or caffeoylquinic acids were found to be the main contributors to the free radical-scavenging activity of the TE. Lipid peroxidation, induced with ascorbate/Fe ${ }^{2+}$, was significantly reduced in the presence of the $\mathrm{TE}\left(\mathrm{EC}_{50}=26 \mu \mathrm{g} \mathrm{dwb} / \mathrm{ml}\right)$ and fractions containing flavonoids and/or caffeoylquinic acids. The fraction containing flavonoid aglycones was found to be responsible for a major part of the TE protection against lipid peroxidation. Hypericins and hyperforins made no significant contributions to the antioxidant properties of TE. Human consumption of $H$. perforatum extract or fractions could be beneficial.
\end{abstract}

(c) 2004 Elsevier Ltd. All rights reserved.

Keywords: Hypericum perfortum; Phenolics; Flavonoids; Antioxidant activity; Lipid peroxidation inhibition

\section{Introduction}

Reactive oxygen species (ROS) are generated by many redox processes that normally occur in the metabolism of aerobic cells. These species are highly reactive and harmful to the cells. If not eliminated, ROS can damage important molecules, such as proteins, DNA, and lipids. Cells express several defence mechanisms, including antioxidant enzymes and nonenzymatic compounds, that help prevent the damaging effects of ROS (Ben-Yoseph, Boxer, \& Ross, 1996; Fridovich, 1997). However, these endogenous systems are often in sufficient for complete scavenging of ROS. Their excess has

Abbreviations: BHT, butylated hydroxytoluene; DPPH, 1, 1-diphen- yl-2-picrylhydrazyl; dwb, dry weight biomass; MDA, malondialdehyde; TBA, thiobarbituric acid; TBARS, thiobarbituric acidreactive species; TE, Hypericum perforatum total ethanolic extract.

${ }^{*}$ Corresponding author. Tel.: +351-253-604318; fax: +351-253678980.

E-mail address: acpdias@bio.uminho.pt (A.C.P. Dias). been implicated in the development of chronic diseases, such as cancer, arteriosclerosis and rheumatism (Halliwell, 1996, 1999). Oxidative stress can also play an important role in the development of neurodegenerative disorders, such as Alzheimer's and Parkinson's diseases (Behl \& Mosmann, 2002; Foy, Passmore, Vahidassr, Young, \& Lawson, 1999).

There is an increasing interest in natural antioxidants, namely phenols, present in medicinal and dietary plants, that might help prevent oxidative damage (Gardner, White, McPhail, \& Duthie, 2000; Halliwell, 1996, 1999; Youdim, Spencer, Schroeter, \& Rice-Evans, 2002; Zheng \& Wang, 2001). In situations of increased free radical generation the reinforcement of endogenous antioxidants via intake of dietary antioxidants may be of particular importance in attenuating the cumulative effects of oxidatively damaged molecules.

In recent years, the consumption of Hypericum perforatum (St. John's wort)-derived products has increased dramatically and presently it is one of the most 
consumed medicinal plants over the world (Wills, Bone, \& Morgan, 2000). The commercially available $H$. perforatum derived products include sophisticated phytopharmaceuticals and nutraceuticals, teas, tinctures, juices and oily macerates (Gaedcke, 2003). Also, several brands of common food products, such as beverages and yoghurts, include St John's wort extracts as additives.

$H$. perforatum has a wide range of medicinal applications, including skin wounds, eczema, burns, diseases of the alimentary tract and psychological disorders. Nowadays, its use in the treatment of mild to moderate depression has become prominent (Butterweck, 2003; Di Carlo, Borrelli, Ernst, \& Izzo, 2001; Erdelmeier, Koch, $\&$ Hoerr, 2000). Numerous papers have been published concerning these aspects and several recent reviews can be pointed out (Agostinis, Vantieghem, Merlevede, \& de Witte, 2002; Butterweck, 2003; Di Carlo et al., 2001; Erdelmeier et al., 2000; Prince et al., 2001). In spite of this intense research activity, the antioxidant potential of $H$. perforatum extracts has been somewhat neglected. St. John's wort medicinal properties, namely those regarding the antidepressant activity, have been related to their phenolic composition and particularly hypericins, hyperforins and flavonoids (Butterweck, 2003). H. perforatum ethanolic extracts contain many phenolic compounds, namely flavonoids and phenolic acids, suggesting that they could have important antioxidant properties.

Data on the antioxidant properties of $H$. perforatum extracts are scarce. Previous reports indicate that these extracts show significant scavenging capacity for the superoxide radical, produced by the xanthine/xanthine oxidase system (Hunt, Lester, Lester, \& Tackett, 2001). Nevertheless, no correlation between these properties and the phenolic composition of the extracts has been investigated. The aim of this study was to evaluate antioxidant potential of several $H$. perforatum extracts and to determine its relationship with the phenolic composition, by analytical procedures.

\section{Materials and methods}

\subsection{Compounds}

Chlorogenic acid, quercetin, rutin, DPPH, BHT, $\alpha$ tocopherol (Trolox) and thiobarbituric acid were purchased from Sigma (Barcelona, Spain). Hyperoside, isoquercetin, kaempferol, amentoflavon, and hypericin were obtained from Extrasynthése (Genay, France). Hyperforin tetramonium salt was a gift from Dr. Clemans Erdelmeier (Wilmar Schwabe GmbH, Germany). Sephadex LH-20 resin was supplied by Pharmacia (Uppsala, Sweden). All other reagents were of analytical grade and supplied by Merck (Darmstadt, Germany).
2.2. Plant material and production of extracts of $H$. perforatum

H. perforatum plants (flowers, including approx. $7 \mathrm{~cm}$ of stem) were collected in the region of Braga, North of Portugal, and the biomass was freeze-dried on the same day. An ethanolic extract was prepared by extraction of the biomass $(100 \mathrm{mgdwb} / \mathrm{ml})$ with an ethanol-water solution (80:20). The mixture was filtered through a paper filter (Whatman, No. 1) and the resulting total ethanolic extract of $H$. perforatum (TE) was stored in a dark glass bottle for further processing.

An aliquot of TE was sequentially fractionated by liquid-liquid extraction with hexane, ethyl acetate, and butanol. The hexane, ethyl acetate and butanol extracts were evaporated, separately, under reduced pressure, at $35^{\circ} \mathrm{C}$ in darkness. Hexane and butanol extracts residues were named, respectively, fractions V1 and V6. The ethyl acetate residue was dissolved in methanol and fractionated over Sephadex LH-20 (column: $40 \times 3 \mathrm{~cm}$ ), using methanol as eluent. The extracts resulting from column elution were analysed by TLC, grouped according their chemical composition, and evaporated under reduced pressure, as described above, resulting in the fractions V2-V5. TLC was performed on silica gel aluminium plates using three different eluents for each sample. Eluents utilised were ethyl acetate:formic acid:glacial acetic acid:water (100:11:11:27), toluene:chloroform:acetone:formic acid (40:25:35:1), and toluene:ethyl acetate:formic acid (6:4:1). The aqueous extract remaining from the sequential liquid-liquid extraction of TE was lyophilised, resulting in a pale-cream powder named fraction V7. All fractions were dissolved in ethanol, and a small aliquot was taken for HPLC analysis. The remaining material was stored at $-80^{\circ} \mathrm{C}$, under a nitrogen atmosphere, prior to further experiments.

\subsection{Phenolic identification and quantification}

TE and fractions were analysed by HPLC-DAD as described previously (Dias, Seabra, Andrade, \& Ferreira, 1999). Chromatograms were recorded at 260, 350 and $590 \mathrm{~nm}$. The quantification of the constituents was done by the external standard method, using a solution containing $15 \mu \mathrm{g} / \mathrm{ml}$ of each reference compound in methanol. The reference compounds were chlorogenic acid, rutin, hyperoside, isoquercitrin, quercetin, kaempferol, amentoflavone, hypericin and hyperforin salt. Flavonols, flavones and caffeoylquinic acids were quantified at $350 \mathrm{~nm}$, hyperforins at $260 \mathrm{~nm}$ and hypericins at $590 \mathrm{~nm}$. Other flavonols and cinnamic acidtype compounds were quantified at $350 \mathrm{~nm}$, as quercetin and chlorogenic acid equivalents, respectively. Adhyperforin and hypericins were quantified at 260 and 590 $\mathrm{nm}$, as hyperforin and hypericin equivalents, respectively. All the samples were analysed in triplicate. 
Phenolic identification was also performed by HPLC-MS-MS. Chromatographic separation was carried out on an RP C18 column $(25 \times 0.4 \mathrm{~cm}$, particle size $5 \mu \mathrm{m}$, Merck, Germany), using water/formic acid (99:1) and methanol as the mobile phases. Elution was performed as described elsewhere (Dias et al., 1999). The HPLC system was an Agilent HPLC 1100 instrument series equipped with an Agilent DAD detector (G1315B, Agilent Technologies, Germany) and mass detector in series, controlled by software from Agilent Technologies (Germany). The mass detector was an ion-trap mass spectrometer (G2445A, Agilent Technologies, Germany) equipped with an electrospray ionization (ESI) system. The heated capillary and voltage were maintained at $350{ }^{\circ} \mathrm{C}$ and $4 \mathrm{kV}$, respectively. The nebulizer pressure and flow rate of nitrogen were $65.0 \mathrm{psi}$ and $11 \mathrm{l} /$ min, respectively. Mass scan (MS) and daughter (MSMS) spectra were measured from 100 au to $m / z 1500$. Collision-induced fragmentation experiments were performed in the ion trap using helium as the collision gas, with voltage ramping cycles from 0.3 up to $2 \mathrm{~V}$. Mass spectrometry data were acquired, both in the negative and positive modes.

\subsection{Free radical-scavenger activity}

The free radical-scavenger activity was determined by the 1,1-diphenyl-2-picrylhydrazyl (DPPH) assay, as described previously (Silva \& Dias, 2002). The antiradical activity of each fraction was evaluated using a dilutions series, in order to obtain a large spectrum of sample concentrations. Additionally, the relative free radicalscavenging activity was assessed using fractions with equivalent dry-weight biomass concentrations. The reaction solution consisted of $0.1 \mathrm{ml}$ of sample and $1.4 \mathrm{ml}$ of a DPPH stock solution $(80 \mu \mathrm{M}$, ethanol $100 \%)$. The absorbance was monitored continuously at $515 \mathrm{~nm}$ with a Perkin-Elmer UV/VIS Spectrometer Lambda2, assuring that the reaction was complete (plateau state). Ethanol was used as a blank and trolox $(0.5 \mathrm{mg} / \mathrm{ml})$ was used as a positive control. All determinations were performed in triplicate.

The percentage of reduced DPPH at steady state (DPPH R) was calculated and these values were plotted against the $\log _{10}$ of the concentrations of individual fractions. A decrease by $50 \%$ of the initial DPPH concentration was defined as the $\mathrm{EC}_{50}$. The amount of reduced DPPH was estimated for the first $5 \mathrm{~min}$ of reaction for each fraction, at the dilution factor closest to the estimated value $\mathrm{EC}_{50}$. These values were converted to a ratio by dividing them by total DPPH reduced at the steady state. This ratio was named reduction index $\left(\mathrm{RIt} 5^{\prime}\right)$, a kinetic parameter that measures the velocity of the reaction of DPPH reduction. All the parameters were calculated graphically using the software GraphPad 4.0 (Prism, USA).

\subsection{Evaluation of lipid peroxidation - TBARS assay}

The extent of lipid peroxidation was evaluated by measuring the production of TBARS in the cortical synaptosomes of male Wistar rat brains (1.5 months old). The synaptosomal fraction was isolated as previously described (Silva, Carvalho, Carvalho, \& Malva, 2001). The thiobarbituric acid assay was performed as described elsewhere (Pereira, Moreira, Seica, Santos, \& Oliveira, 2000), with minor modifications. The synaptosomal fraction was diluted to obtain a final concentration of $0.5 \mathrm{mg}$ protein $/ \mathrm{ml}$. Lipid peroxidation was assayed after incubation of synaptosomes with $800 \mu \mathrm{M}$ ascorbic acid and $2.5 \mu \mathrm{M} \mathrm{FeSO}_{4}$, in a final volume of 1.2 $\mathrm{ml}$ of Krebs buffer $(132 \mathrm{mM} \mathrm{NaCl}, 4 \mathrm{mM} \mathrm{KCl}, 1.4 \mathrm{mM}$ $\mathrm{MgCl}_{2}, 1 \mathrm{mM} \mathrm{CaCl}, 6 \mathrm{mM}$ glucose, $10 \mathrm{mM}$ Hepes-Na,

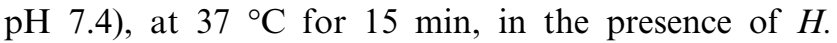
perforatum fractions, with equivalent dry-weight biomass. Controls were made with synaptosomes, without the oxidant pair. BHT $(0.45 \mathrm{mM}$, ethanol $100 \%)$ was used as a positive control. The colour of the (MDA)TBA complex was detected at $530 \mathrm{~nm}$, after reaction of samples with TCA $(40 \%, \mathrm{w} / \mathrm{v})$ and TBA $(0.67 \%$, w/v) $\left(100{ }^{\circ} \mathrm{C}, 10 \mathrm{~min}\right)$ and centrifugation $(3000 \mathrm{~g}, 10 \mathrm{~min})$. The amount of MDA formed was calculated using the absorption coefficient of $1.56 \times 10^{5} \mathrm{~cm}^{2} / \mathrm{mol}$. Values were corrected for the levels of basal peroxidation, obtained during the assay.

The percentage of inhibition of lipid peroxidation (after $15 \mathrm{~min}$ of reaction) was calculated as the reduction in the levels of lipid peroxidation induced by the oxidant pair, after correction for the basal levels (here considered as $100 \%$ ). These values were plotted against the $\log _{10}$ of the concentrations of individual fractions. A decrease by $50 \%$ of the value of lipid peroxidation, induced by ascorbate $/ \mathrm{Fe}^{2+}$, was defined as the $\mathrm{EC}_{50}$.

\subsection{Statistical analysis}

All data was analysed statistically with Statistica/Mac software (Prism, USA). Mean differences were analysed statistically by running a one way analysis of variance test (ANOVA). The homogeneity of variance was tested by Cochran's test. Post-hoc comparisons were made with the HSD Tukey test.

\section{Results and discussion}

\subsection{Fractionation and phenolic composition of the extracts}

Several papers have reported the analysis of $H$. perforatum extracts. However, most of them focus only on some individual compounds, such as the hypericins or the phloroglucinols; LC-MS studies were also done 
(Fuzzati, Gabetta, Strepponi, \& Villa, 2001; Tolonen, Uusitalo, Hohtola, \& Jalonen, 2002). In this work, we fully analysed the TE by HPLC-DAD and HPLCDAD-MS-MS and the major compounds were identified and characterised. Phenolic compounds were identified by their UV spectra and by their MS spectra, and their corresponding daughter MS/MS fragments. TE was composed of a complex mixture of compounds (Fig. 1), most of them already known to be present in $H$. perforatum extracts (Dias et al., 1999; Erdelmeier et al., 2000; Jürgenliemk \& Nahrstedt, 2002), and two compounds being identified for the first time in $H$. perforatum extracts. Table 1 shows the MS data obtained by LC-MS of the most representative phenolics present in TE.

Compounds 1, and 3 have similar UV-spectra, characteristic of chlorogenic-type acids, and have the same deprotonated molecular ion $(353.3, \mathrm{~m} / \mathrm{z})$, indicating that they are isomers. These compounds were identified as 3-O-caffeoylquinic and 5-O-caffeoylquinic acids, respectively, according with these described elsewhere (Dias et al., 1999). HPLC-MS data confirmed the identification. Compound 2 is a derivative of $p$-coumaric acid accordingly to its UV-Vis and MS data. Probably, it corresponds to 3-O-p-coumaroyl quinic acid, a compound already detected in $H$. perforatum extracts (Jürgenliemk \& Nahrstedt, 2002).

A major group of compounds was identified as flavonols, due to their characteristic UV spectra. Compounds 4 and 5 have UV-spectra characteristic of flavonols glycosylated at C3 $(257,265 \mathrm{sh}, 355 \mathrm{~nm})$. Compound 4 has a molecular ion $m / z[\mathrm{M}-\mathrm{H}]^{-}$of 609.0, and its $-\mathrm{MS}^{2}[\mathrm{M}-\mathrm{H}]^{-}$gave a single ion at $\mathrm{m} / \mathrm{z} 300.4$ (Table 1), indicating that it is a quercetin derivative with a rhamnoglucoside at $\mathrm{C} 3$. The absence of intermediate fragmentation between the deprotonated molecular ion and the aglycone ion is indicative of an interglycosidic linkage $1 \rightarrow 6$ (Cuyckens, Rozenberg, Hoffmann, \& Claeys, 2001); therefore this compound was putatively identified as rutin. Compounds 5 has a molecular ion $\mathrm{m} / \mathrm{z}[\mathrm{M}-\mathrm{H}]^{-}$of 462.9 , and its $-\mathrm{MS} 2[\mathrm{M}-\mathrm{H}]^{-}$gave an ion at $\mathrm{m} / \mathrm{z} 300.4$ (Table 1), indicating that this compound is a quercetin derivative with a hexose at $\mathrm{C} 3$, probably hyperoside. The utilization of rutin, hyperoside commercial standards confirmed the identification of compounds 4 and 5. Compounds 7 and 8 shared the same UV-spectra, similar to those observed for flavonols 4 and 5. Both compounds have a similar deprotonated molecular ion $\mathrm{m} / \mathrm{z}$ at 477.3 and a major - $\mathrm{MS}^{2}$ fragment at $\mathrm{m} / \mathrm{z} 300.5$, corresponding to the loss of a glucuronic or a galacturonic acid residue (Table 1). Therefore, these compounds were identified as quercetin 3-glucuronate and quercetin 3-galacturonate, respectively. Compound 9 has an UV-spectrum $(255,265 \mathrm{sh}, 301 \mathrm{sh}, 349)$ and MS data consistent with those of quercetin 3-rhamnoside. The identification was confirmed by spiking with a commercial standard of quercetin 3-rhamnoside and confirmation of its MS data. Compound 10 was identified as a kaempferol derivative, due to its characteristic UV-spectra $(267,297 \mathrm{sh}, 349 \mathrm{~nm})$, with substitution at position 3. The -MS and - $\mathrm{MS}^{2}$ spectra were consistent with the presence of a rutinoside residue (Cuyckens et al., 2001), and confirm the kaempferol aglycone, this compound being identified as kaempferol 3-rutinoside. Compound 11 hold a similar UV-spectrum to those of compounds 4, 5, 6 and 7, indicating its quercetin 3-derivative nature. It has a deprotonated molecular ion at $\mathrm{m} / \mathrm{z} 505.2$ and a $-\mathrm{MS}^{2}$ fragment with an $\mathrm{m} / \mathrm{z}$ of 462.7 (quercetin 3-galactoside/glucoside), that results from the loss of an acetyl group. Moreover, an intense deprotonated ion of the aglycone was observed $(\mathrm{m} / \mathrm{z} 300.2)$. According to its UV and mass spectra this compound is an acetyl derivative of hyperoside or isoquercitrin, probably quercetin-3-O-( $2^{\prime \prime}$-acetyl)- $\beta$ - $D$-galactoside, a compound recently identified in $H$. perforatum extracts (Jürgenliemk \& Nahrstedt, 2002). Compound 12 had a similar UV-spectrum to compounds 5 and 11 , a $[\mathrm{M}-\mathrm{H}]^{-}$ ion at $\mathrm{m} / \mathrm{z}$ 651.7, and $-\mathrm{MS}^{2}[\mathrm{M}-\mathrm{H}]^{-}$ions at $\mathrm{m} / \mathrm{z} 608.6$ (M-H-Acetyl), corresponding to the loss of an acetyl group from the deprotonated molecular ion, and at $\mathrm{m} / \mathrm{z}$ 300.2, indicating its rutin-derivative nature. Compounds 10 and 12 are now reported for the first time in $H$. perforatum extracts. Compound 13 has a UV-spectrum $(255,265 \mathrm{sh}, 370 \mathrm{~nm})$ and MS data consistent with those of quercetin. The identification was confirmed by spiking with a commercial standard of quercetin and confirmation of its MS data. Compound 15 has a UVspectrum $(268,333 \mathrm{~nm})$, similar to that of amentoflavone, and a $[\mathrm{M}-\mathrm{H}]^{-}$ion at $\mathrm{m} / \mathrm{z} 537.4$. These data and its localization in the chromatogram are compatible with biapigenin.

The compounds 17-20 were identified as hypericins according to their characteristic UV-Vis spectra (Kurth \& Spreemann, 1998). Confirmation of hypericin identity was supported by MS data consistent with that already published (Tolonen et al., 2002). Hyperforin and adhyperforin were identified as described elsewhere (Dias \& Ferreira, 2003), and MS data agreed with published reports (Fuzzati et al., 2001; Tolonen et al., 2002).

The fractions of $H$. perforatum were separated by chromatographic procedures and the compounds present in each fraction were identified and quantified by HPLC-DAD and HPLC-MS. The results of quantitative analysis by HPLC-DAD are presented in Table 2 . These results were expressed in $H$. perforatum biomass equivalents since, in this way, all the fractions were easily compared with the TE.

All compounds identified in the TE were specifically concentrated in the individual fractions: fraction V1 contains derivatives of acylphloroglucinols, mainly hyperforin; fraction V2 contains hypericin and some related compounds (bianthraquinones); fraction V3 is 

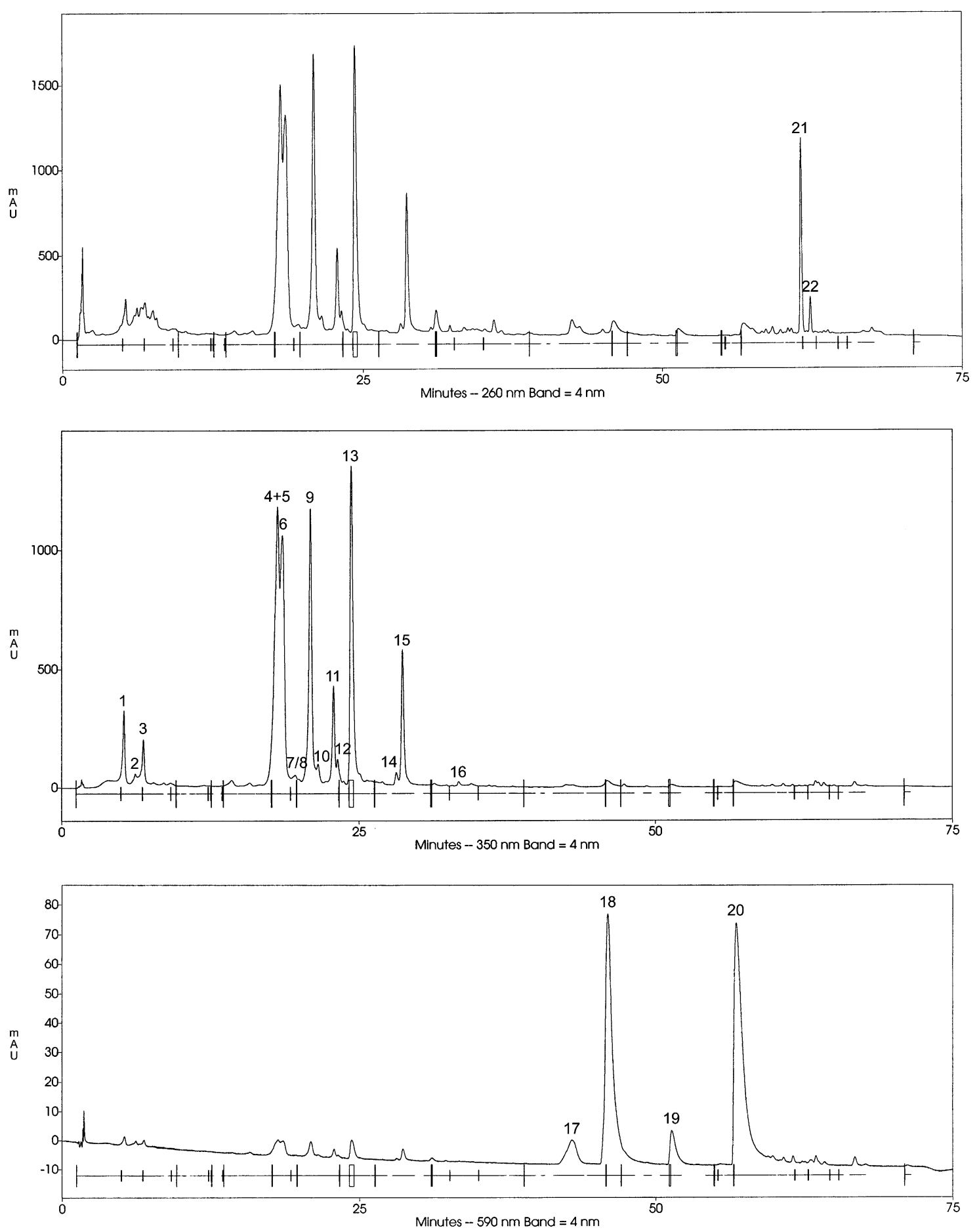

Fig. 1. HPLC chromatogram of $H$. perforatum total ethanolic extract (TE). Compounds are identified in the figure by numbers: $1-$ neochlorogenic acid (3-O-caffeoylquinic acid); 2 - caffeoylquinic acid type; 3 - chlorogenic acid (5-O-caffeoylquinic acid); 4 - rutin (quercetin-3-O-rutinoside); 5 - hyperoside (quercetin-3-O- $\beta$-D-galactoside); 6 - isoquercetin (quercetin-3-O- $\beta$-D-glucoside); 7 - quercetin 3 - $O$ - $\beta$-D-galacturonic; 8 - quercetin 3 O- $\beta$-D-glucuronic; 9 - quercitrin (quercetin 3-O - $\alpha$-L-rhamnoside); 10 - kaempferol 3-O- $\beta$-D-rutinoside; 11 - hyperoside-acetyl; 12 - rutin-acetyl; 13 - quercetin; 14 - kaempferol; 15 - biapigenin; 16 - amentoflavone; 17 - protopseudohypericin; 18 - pseudohypericin; 19 - protohypericin; 20 hypericin; 21 - hyperforin; 22 - adhyperforin.

composed of a mixture of cinnamic acids and flavonol glycosides, the former occurring in higher amounts; fraction V4 is composed solely of flavonol glycosides, mainly of the quercetin-type; fraction V5 is composed of flavonoid aglycones, namely quercetin and biapigenin; fraction V6 is composed of caffeoylquinic acids and glycosides of flavonoids, with the latter occurring in higher amounts; fraction V7 contains several 
Table 1

HPLC-MS-MS data of the major identified compounds of $H$. perforatum

\begin{tabular}{cllc}
\hline Compound & {$[\mathrm{M}-\mathrm{H}]^{-}(\mathrm{m} / \mathrm{z})$} & $-\mathrm{MS}^{2}[\mathrm{M}-\mathrm{H}]^{-}(\mathrm{m} / z)$ & $R t(\mathrm{~min})$ \\
\hline 1 & 353.3 & & 5.25 \\
2 & 337.1 & & 6.20 \\
3 & 353.3 & & 6.88 \\
4 & 609.0 & 300.4 & 17.95 \\
5 & 462.9 & 300.4 & 18.15 \\
7 & 477.2 & 300.5 & 18.58 \\
8 & 477.3 & 300.5 & 19.54 \\
9 & 446.6 & 300.3 & 19.93 \\
10 & 593.5 & 284.4 & 20.92 \\
11 & 505.2 & $462.7,300.2$ & 22.87 \\
12 & 651.7 & $608.6,590.5,300.2$ & 23.22 \\
13 & $302.6^{\mathrm{a}}$ & & 24.35 \\
15 & 537.4 & $442.5,384.7$ & 28.68 \\
17 & 521.3 & 423.1 & 42.91 \\
18 & 519.1 & $487.4,421.2$ & 45.96 \\
19 & 505.3 & 407.2 & 51.36 \\
20 & 503.3 & 405.3 & 56.80 \\
21 & 535.2 & 383.1 & 61.59 \\
22 & 549.9 & 397.3 & 62.36 \\
\hline
\end{tabular}

$R t$ - retention time in high-precision liquid chromatography; compound numbers correspond to those indicated in Fig. 1.

${ }^{\mathrm{a}}$ Ion obtained in the positive mode, corresponding to the protonation $[\mathrm{M}+\mathrm{H}]^{+}$.

compounds of different phenolic classes, present at low concentrations.

The results indicate that the fractionation process resulted in extracts that, in global terms, contained similar amounts of the compounds present in the original extract (TE) (Table 2). A significant exception was observed for hypericins. The amount of pseudohypericin found in fraction V2 was lower than that observed in the TE. This could be due to a selective absorption of pseudohypericin on the Sephadex resin.

\subsection{Antioxidant properties of $H$. perforatum extracts}

In this work, the antioxidant activities of TE and isolated fractions were examined by two model systems, the DPPH method and the inhibition of lipid peroxidation in synaptosomes.

DPPH is a useful reagent for investigating the free radical-scavenging activities of phenolic compounds (Lebeau et al., 2000). The reduction of DPPH absorption is indicative of the capacity of the extracts to scavenge free radicals, independently of any enzymatic activity. The extraction methods resulted in different extracts, with diverse compositions, either in qualitative or quantitative terms (Table 2). We chose to normalize the results in terms of plant dry weight biomass equivalents, so that the TE and all fractions could be related to the biomass used, making it easier to compare the relative importance and potency of each fraction for scavenging the DPPH radical. TE and all fractions promoted the reduction of DPPH (Table 3). The best free radical-scavenger activity was reported for the TE and fractions V4 and V6, with $\mathrm{EC}_{50}$ values of 21, 80 and $82 \mathrm{~g} \mathrm{dwb} / \mathrm{ml}$, respectively (Table 3 ). The lowest activity was observed for fraction V7. This fraction resulted from the aqueous extract, that remained after the sequential extraction of the TE with organic solvents (see Materials and methods), being a very dilute fraction in terms of compounds per biomass equivalents (Table 2). This can justify the low activity registered for this fraction. Trolox, used as a positive control in this assay, exhibited the highest antiradical activity $\left(\mathrm{EC}_{50}=2.2\right.$ $\mathrm{g}$ compound $/ \mathrm{ml}$ ). However, it noteworthy mentioned that the trolox $\mathrm{EC}_{50}$ value is for a pure compound, whereas the values for the TE and fractions were expressed in amounts of dry weight biomass.

The $\mathrm{EC}_{50}$ is a parameter, widely used, that describes ability of a compound or extract to scavenge the DPPH radical. However, the $\mathrm{EC}_{50}$ values give little information about the kinetics of a reaction, in terms of velocity (Lebeau et al., 2000). Therefore, we used an additional parameter, the reduction index (RIt5'), to complement the information (Table 3). This parameter gives an idea of the reactivity of each fraction in bleaching the DPPH free radical. All fractions induced a moderate decrease in the concentration of DPPH (when compared with trolox reactivity). The most reactive were the TE and fractions V3-V6, exhibiting similar RIt5' values, although with significantly different $\mathrm{EC}_{50}$ values (Table 3 ). It is interesting to note that all these extracts possessed high reactivity and had an important flavonoid component (namely fractions V4 and V5). Fraction V7 exhibited the weakest reactivity, significantly different from any other extract, in spite of having a considerable proportion of flavonoids and caffeoylquinic-type acids. This low reactivity could be related to the presence of other compounds (e.g. sugars, amino acids), not detected by the HLPC procedures, that could somehow interfere in the total reactivity of the fraction.

We evaluated the extent of lipid peroxidation using the MDA formation as an index of breakdown of membrane lipids, after incubation of rat cortical synaptosomes with the oxidant pair ascorbate $/ \mathrm{Fe}^{2+}$. Brain homogenate is useful for the investigation of lipid peroxidation and assessment of oxidative stress. Ferrous ion stimulates lipid peroxidation of brain homogenate through various mechanisms, for example through the decomposition of lipid peroxides, the generation of hydroxyl radicals, or by formation of perferryl or ferryl species (Ko, Cheng, Lin, \& Teng, 1998).

Lipid peroxidation was assessed in the presence of $H$. perforatum total ethanolic extract (TE). Fig. 2 shows the protection conferred by increasing concentrations of $\mathrm{TE}$, in lipid peroxidation induced by ascorbate $/ \mathrm{Fe}^{2+}$ in rat cortical synaptosomes. TE reduced lipid peroxidation in a dose-dependent manner, and proved to be an 
Table 2

Phenolic composition of H. perforatum extract (TE) and fractions (HPLC-DAD and HPLC-DAD-MS analysis)

\begin{tabular}{|c|c|c|c|c|c|c|c|c|}
\hline \multirow[t]{2}{*}{ Compound } & \multicolumn{8}{|c|}{ Fraction (g compound/g dwb) } \\
\hline & $\mathrm{TE}$ & $\mathrm{V} 1$ & $\mathrm{~V} 2$ & $\mathrm{~V} 3$ & $\mathrm{~V} 4$ & V5 & V6 & V7 \\
\hline \multicolumn{9}{|l|}{ Phloroglucinols } \\
\hline Hyperforin & 7400 & 6569 & & & & & & \\
\hline Adhyperforin & 1470 & 1070 & & & & & & \\
\hline Total & 8870 & 7639 & & & & & & \\
\hline \multicolumn{9}{|l|}{ Naphthodianthrones } \\
\hline Hypericin & 620 & & 871 & & & & & $\operatorname{Tr}$ \\
\hline Pseudohypericin & 839 & & 62 & & & & & $\operatorname{Tr}$ \\
\hline Protopseudohypericin & 79 & & 53 & & & & & \\
\hline Protohypericin & 80 & & 56 & & & & & \\
\hline Total & 1618 & & 1042 & & & & & \\
\hline \multicolumn{9}{|l|}{ Phenolic acids } \\
\hline Neochlorogenic acid & 1880 & & & 1002 & & & 538 & 277 \\
\hline Chlorogenic acid & 1181 & & & 745 & & & 330 & 45 \\
\hline Unindentified & 189 & & & 103 & & & 50 & $\mathrm{Tr}$ \\
\hline Total & 3250 & & & 1850 & & & 918 & 322 \\
\hline \multicolumn{9}{|l|}{ Flavonol glycosides } \\
\hline Hyperoside + Rutin & 5861 & & & 625 & 4050 & & 871 & 290 \\
\hline Isoquercitrin & 2442 & & & 188 & 1589 & & 539 & \\
\hline $\begin{array}{l}\text { Quercetin 3-glucuronate } \\
\quad+\text { Quercetin 3-galacturonate }\end{array}$ & 222 & & & & 170 & & 23 & 10 \\
\hline Hyperoside-acetyl & 888 & & & 83 & 786 & & 21 & 20 \\
\hline Rutin-acetyl & 133 & & & & 98 & & $\operatorname{Tr}$ & $\operatorname{Tr}$ \\
\hline Kampferol 3-rutinoside & 225 & & & & 160 & & 65 & \\
\hline Quercetin 3-rhamnoside & 3159 & & & 97 & 2835 & & 126 & 60 \\
\hline Total & 12,930 & & & 993 & 9688 & & 1645 & 320 \\
\hline \multicolumn{9}{|l|}{ Flavonoid aglycones } \\
\hline Quercetin & 4084 & & & & & 3960 & & $\operatorname{Tr}$ \\
\hline Kaempferol & 256 & & & & & 205 & & $\operatorname{Tr}$ \\
\hline Biapigenin & 1004 & & & & & 934 & & $\operatorname{Tr}$ \\
\hline Amentoflavone & 58 & & & & & $\operatorname{Tr}$ & & \\
\hline Total & 5402 & & & & & 5099 & & \\
\hline Total phenolics & 32,070 & 7639 & 1042 & 2843 & 9688 & 5099 & 2563 & 702 \\
\hline
\end{tabular}

$\mathrm{Tr}$ - compounds at trace levels, detected only by HPLC-MS/MS.

Table 3

Free radical-scavenger activity $\left(\mathrm{EC}_{50}\right)$ and initial reduction kinetics $\left(\mathrm{RIt}^{\prime}\right)$ of $H$. perforatum total ethanolic extract (TE) and fractions

\begin{tabular}{lll}
\hline Sample & $\mathrm{EC}_{50}(\mu \mathrm{g} \mathrm{dwb} / \mathrm{ml})$ & $\mathrm{RIt}^{\prime}$ \\
\hline Total extract (TE) & 21 & $0.68 \mathrm{a}$ \\
V1 & 290 & $0.54 \mathrm{~b}$ \\
V2 & 507 & $0.60 \mathrm{~b}$ \\
V3 & 406 & $0.71 \mathrm{a}$ \\
V4 & $80 \mathrm{a}$ & $0.67 \mathrm{a}$ \\
V5 & 145 & $0.68 \mathrm{a}$ \\
V6 & $82 \mathrm{a}$ & $0.70 \mathrm{a}$ \\
V7 & 870 & 0.36 \\
Trolox & $2^{\mathrm{A}}$ & 0.94 \\
\hline
\end{tabular}

Values are mean means of six independent replicates. In each column, the values were statistically different $(P<0.05)$, except those with the same letter.

${ }^{\mathrm{A}}$ The $\mathrm{EC}_{50}$ values were calculated in terms of dry weight biomass with the exception of trolox, calculated as the amount of pure compound.

effective antioxidant. The $\mathrm{EC}_{50}$ value, determined for the TE inhibitory effect on lipid peroxidation, was 26 $\mu \mathrm{gdwb} / \mathrm{ml}$. This value is in the same range as that

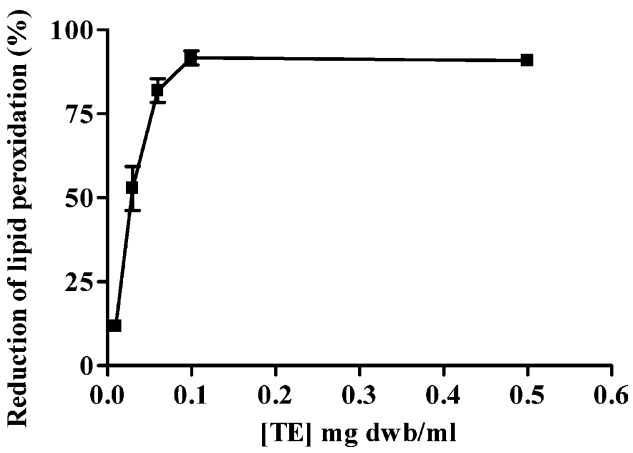

Fig. 2. Inhibitory effect of $H$. perforatum total ethanolic extract (TE) on lipid peroxidation induced by the oxidant pair ascorbate/ $\mathrm{Fe}^{2+}$, in rat cortical synaptosomes. The oxidant pair increased basal levels of peroxidation by approximately 15 -fold. Values are means \pm SD of six independent replicates.

observed for the TE scavenger activity of DPPH (Table 3).

In order to evaluate the relative contribution of each fraction to the total antioxidant activity of the TE (that 
represents the sum of all fractions), we evaluated the free radical-scavenging activity (Table 4 ) and lipid peroxidation inhibition (Table 5) of all fractions on an equivalent plant biomass concentration basis. Fractions V5, V6, and V4, composed of flavonol glycosides, showed the highest free radical-scavenging activity and contributed most of the activity of the TE.

The most effective fraction in protecting synaptosomes from lipid peroxidation was V5, that contained flavonoid aglycones (Table 5). At concentrations of 0.1 $\mathrm{mg} \mathrm{dwb/ml,} \mathrm{fraction} \mathrm{V5} \mathrm{exhibited} \mathrm{a} \mathrm{similar} \mathrm{potency} \mathrm{(in}$ reducing lipid peroxidation), to the TE. Fractions V3, V5, and V6 were able to inhibit lipid peroxidation, with similar efficacy to the TE, only at concentrations of 2 $\mathrm{mgdwb} / \mathrm{ml}$. Fractions V1 and V2, containing respectively hyperforins and hypericins, were ineffective in reducing lipid peroxidation, at all concentrations tested (Table 5).

Among the fractions tested, fractions V4 and V5, composed basically of quercetin glycosides and aglycones, respectively, contributed mainly to the TE antioxidant activity (Tables 4 and 5). Fraction V4 had a stronger free radical-scavenging activity, whereas frac- tion V5 was more effective in protecting against lipid peroxidation. In general, the substitution of hydroxyl groups by glycosylation decreases the antioxidant activity of a phenolic compound (Rice-Evans, Miller, \& Paganga, 1997). Specifically, glycosylation resulted in lower antioxidant activity for quercetin using DPPH (Fukumoto \& Mazza, 2000). Addition of a sugar moiety decreases activity of the aglycone, probably due to steric hindrance. Also, Cos et al. (2001), using a lipid peroxidation model in rat liver microsomes, observed that quercetin had lower $\mathrm{IC}_{50}$ values than its glycosylated derivatives quercitrin and rutin. This is in accordance with the higher lipid peroxidation inhibition observed for fraction V5. However, this does not explain the higher free radical-scavenging activity of fraction V4 than V5. Fraction V4 had a significantly higher amount of total flavonoids (almost 2-fold) than fraction V5 (Table 2). Therefore, in this case the free radical-scavenging effect measured would be more influenced by the high quantity of flavonols present in fraction V4, rather than by the structural characteristics of the compounds. In the lipid peroxidation model, besides the structural effects, other factors, such as the hydrophobicity of the

Table 4

Relative antiradical activity of $H$. perforatum total ethanolic extract (TE) and fractions

\begin{tabular}{|c|c|c|c|c|c|c|c|c|c|c|}
\hline & \multicolumn{10}{|c|}{ Equivalent biomass concentration (mg dwb/ml) } \\
\hline & 0.010 & 0.015 & 0.025 & 0.050 & 0.075 & 0.100 & 0.150 & 0.250 & 0.500 & 1.500 \\
\hline TE & 0.0 & 37.6 & 55.4 & 74.2 & 80.8 & 83.8 & $86.4 \mathrm{a}$ & 88.1ab & $89.1 \mathrm{~b}$ & $89.5 \mathrm{~b}$ \\
\hline V1 & $1.7 \mathrm{a}$ & $1.7 \mathrm{a}$ & $1.7 \mathrm{a}$ & $1.7 \mathrm{a}$ & $1.8 \mathrm{a}$ & $1.9 \mathrm{ab}$ & $2.1 \mathrm{~b}$ & 2.7 & 5.3 & 24.3 \\
\hline V2 & $5.5 \mathrm{a}$ & $5.5 \mathrm{a}$ & $5.5 \mathrm{a}$ & $5.5 \mathrm{a}$ & $5.5 \mathrm{a}$ & $5.5 \mathrm{a}$ & $5.5 \mathrm{a}$ & $5.6 \mathrm{a}$ & 6.2 & 11.6 \\
\hline V3 & $2.6 \mathrm{a}$ & $2.7 \mathrm{a}$ & $2.7 \mathrm{a}$ & $3.0 \mathrm{ab}$ & $3.2 \mathrm{~b}$ & $3.5 b$ & 4.1 & 5.5 & 9.0 & 23.2 \\
\hline V4 & $12.5 \mathrm{a}$ & $12.5 \mathrm{a}$ & $12.5 \mathrm{a}$ & $12.6 \mathrm{a}$ & $12.8 \mathrm{a}$ & $13.1 \mathrm{ab}$ & $14.0 \mathrm{~b}$ & 17.1 & 30.0 & 71.5 \\
\hline V5 & $3.6 \mathrm{a}$ & $3.7 \mathrm{a}$ & $3.7 \mathrm{a}$ & $4.0 \mathrm{ab}$ & $4.4 b c$ & $4.8 \mathrm{c}$ & 5.8 & 8.3 & 16.1 & 46.0 \\
\hline V6 & $5.5 \mathrm{a}$ & $5.6 \mathrm{a}$ & $5.6 \mathrm{a}$ & $5.9 \mathrm{ab}$ & $6.2 \mathrm{bc}$ & $6.7 \mathrm{c}$ & 7.8 & 10.6 & 20.1 & 54.1 \\
\hline V7 & $8.0 \mathrm{a}$ & $8.1 \mathrm{a}$ & $8.1 \mathrm{a}$ & $8.1 \mathrm{a}$ & $8.1 \mathrm{a}$ & $8.1 \mathrm{a}$ & $8.2 \mathrm{a}$ & $8.3 \mathrm{ab}$ & $8.7 \mathrm{~b}$ & 11.5 \\
\hline Sum* & $39.3 \mathrm{a}$ & $39.6 \mathrm{a}$ & $39.8 \mathrm{a}$ & $40.7 \mathrm{ab}$ & $42.0 \mathrm{bc}$ & $43.5 \mathrm{c}$ & 47.4 & 58.1 & 95.6 & 242 \\
\hline
\end{tabular}

Values are in percentages of DPPH reduction and are means of six independent replicates. In each row, all values are statistically different $(P<0.05)$ except those marked with the same letter.

* The row with an asterisk shows the sum of the DPPH reductions of all fractions, except TE, for a given equivalent biomass.

Table 5

Inhibition of lipid peroxidation (\%) of H. perforatum total ethanolic extract (TE) and fractions in lipid peroxidation

\begin{tabular}{|c|c|c|c|c|}
\hline \multirow[t]{2}{*}{ Sample } & \multicolumn{4}{|c|}{ Equivalent biomass concentration (mg dwb/ml) } \\
\hline & 0.03 & 0.1 & 2 & 10 \\
\hline TE & $52.7 \pm 6.59$ & $91.6 \pm 2.07 \mathrm{c}$ & $95.2 \pm 0.57 \mathrm{ac}$ & $95.0 \pm 0.07 \mathrm{ac}$ \\
\hline $\mathrm{V} 1$ & $0.0 \pm 1.04 \mathrm{ac}$ & $0.1 \pm 0.12 \mathrm{ad}$ & $0.1 \pm 2.37 \mathrm{ad}$ & $0.0 \pm 0.15 \mathrm{af}$ \\
\hline $\mathrm{V} 2$ & $0.0 \pm 2.00 \mathrm{ac}$ & $0.0 \pm 0.29 \mathrm{ad}$ & $0.2 \pm 1.66 \mathrm{ad}$ & $0.0 \pm 0.18 \mathrm{af}$ \\
\hline V3 & $0.0 \pm 3.50 \mathrm{ac}$ & $3.1 \pm 2.00 \mathrm{ae}$ & $97.9 \pm 1.07 \mathrm{c}$ & $23.3 \pm 1.72 \mathrm{de}$ \\
\hline V4 & $0.0 \pm 2.15 \mathrm{ac}$ & $3.6 \pm 2.99 \mathrm{ae}$ & $97.8 \pm 0.38 \mathrm{c}$ & $75.4 \pm 6.67$ \\
\hline V5 & $2.2 \pm 5.06 \mathrm{c}$ & $98.9 \pm 5.51 \mathrm{ac}$ & $100.8 \pm 0.20 \mathrm{bc}$ & $99.5 \pm 1.35 \mathrm{abc}$ \\
\hline V6 & $0.0 \pm 5.08 \mathrm{ac}$ & $0.0 \pm 4.37 \mathrm{ad}$ & $96.7 \pm 0.89 \mathrm{c}$ & $27.7 \pm 1.66 \mathrm{~d}$ \\
\hline V7 & $0.0 \pm 2.53 \mathrm{ac}$ & $0.0 \pm 0.21 \mathrm{ad}$ & $48.6 \pm 6.65$ & $20.2 \pm 5.68 \mathrm{e}$ \\
\hline
\end{tabular}

Lipid peroxidation is shown as percentages of reduction, relatively to the levels obtained with the oxidant pair ascorbate/Fe $\mathrm{Fe}^{2+}$. The oxidant pair increased basal levels of peroxidation by approximately 15 -fold. Values are means \pm SD of three to six independent replicates. In each row, all values are statistically different $(P<0.05)$ except those marked with the same letter. In each column, all values are statistically different $(P<0.05)$ except those marked with the same symbol. 
compounds, should be taken into account (Areias, Rego, Oliveira, \& Seabra, 2001). The higher efficiency of flavonoid aglycones in lipid systems, relatively to their glycoside counterparts, is mainly due to their lower polarity and higher partition coefficient (Schroeter, Williams, Matin, Iversen, \& Rice-Evans, 2000). Therefore, the aglycones present in fraction V5 would have a higher ability to interact with the synaptosomal membranes, thus being more effective in preventing lipid peroxidation than quercetin glycoside derivatives, compounds with a more hydrophilic character.

At high biomass equivalents concentrations (10 $\mathrm{mg} \mathrm{dwb/ml),} \mathrm{fractions} \mathrm{V3,} \mathrm{V4,} \mathrm{V6,} \mathrm{and} \mathrm{V7,} \mathrm{exhibited} \mathrm{a}$ pro-oxidant behaviour, shown by decreased efficiency in inhibiting lipid peroxidation (Table 5). A seemingly prooxidant effect was also observed in a cell-free system, using the xanthine/xanthine oxidase system, when an $H$. perforatum extract was tested at high concentrations (Hunt et al., 2001). In our case, the TE did not show any pro-oxidant effect at the concentrations tested, for either antioxidant model (Tables 4 and 5). Some phenolics are capable of redox cycling of the metal ion required for hydroxyl generation, thus increasing the radical production and exhibiting a pro-oxidant activity (Li \& Xie, 2000; Miranda et al., 2000). Valentao et al. (2002) observed that an $H$. androsaemum lyophilised infusion was an effective substitute for ascorbic acid, when at high concentrations and that it could act as a pro-oxidant.

Hunt et al. (2001) evaluated the antioxidant effect of preparations of $H$. perforatum extracts against the superoxide anion. They observed a free radical-scavenging effect for most of the concentrations tested and postulated that this effect could be related to its content in hypericin, among other compounds. Our results demonstrated that compounds other than hypericins should be relevant for this activity. Taking in account the activity of the $H$. perforatum fractions tested, the hydroxycinnamic acids and flavonoids were highly relevant for both radical-scavenging and inhibition of lipid peroxidation activities (Tables 3-5). Conforti, Statti, Tundis, Menichini, and Houghton (2002) evaluated the antioxidant potential of $H$. triquetrifolium Turra in a lipid peroxidation assay. In accordance with our results, they observed that the antioxidant activity of this species could be related to the content of flavonoids. The antioxidant activity of an $H$. androsaemum infusion was also related to its phenolic content (Valentao et al., 2002). Some of the identified phenols in the $H$. androsaemum infusion extract were 3- and 5-O-caffeoylquinic acids, quercetin and glycosylated derivatives, also present in extracts of $H$. perforatum. Flavonoids, specifically quercetin and its glycoside derivatives, are a major class of compounds present in the $H$. perforatum total ethanolic extract (TE), representing almost $57 \%$ of the total phenols present in TE, whereas hydroxycinnamates represent up to $10 \%$ (Table 2). These types of com- pounds are well known antioxidants. They have structural aspects, such as the presence of a catechol moiety in the ring, a 2,3-double bond in conjunction with a 4-oxo group in the C-ring, and the presence of hydroxyl groups at positions 3 and 5, which are all determinants of high antioxidant activity (Halliwell, Aeschbach, Löliger, \& Aruoma, 1995; Rice-Evans et al., 1997).

The observed antioxidant properties of the total ethanolic extract of $H$. perforatum could be, in part, responsible for some of the medicinal claims that are attributed to this plant, namely those related to free radical generation, such as the anti-inflammatory properties. ROS have been proven to cause damage to central nervous systems (Behl \& Mosmann, 2002; Foy et al., 1999; Hyun, Lee, Halwell, \& Jenner, 2002; Markesbery, 1997), so antioxidant properties of products that exert their effects in the CNS, such as $H$. perforatum extracts, could prove to be beneficial in oxidative stress situations at this level. Recently, it was reported that amentoflavone, also identified in our study, is able to pass the blood-brain barrier by simple diffusion (Gutmann et al., 2002). Also, utilizing an in vitro model, it was shown that several dietary flavonoids and their known physiologically relevant metabolites were able to cross the blood-brain barrier (Youdim et al., 2003). So, it seems plausible to assume that the consumption of extracts of $H$. perforatum might be able to exert a positive action in the CNS.

\section{Conclusions}

In conclusion, the total ethanolic extract of $H$. perforatum (TE) possesses high antioxidant activities. The consumption of TE and, certain fractions, either as pharmaceutical preparations or included in food products, has the potential to confer antioxidant properties with benefit to health. It seems that the use of $H$. perforatum extracts cause no apparent significant side effects in most consumers (Kasper, 2001). However, the intake of these extracts together with some conventional drugs, can lead to negative interactions, with undesirable consequences (LaFrance et al., 2000). More scientific work has to be done in order to verify the antioxidant effect of $H$. perforatum extracts in in vivo conditions, and to ensure their effectiveness and absence of adverse effects.

\section{Acknowledgements}

Hyperforin salt was a kind donation of Dr. Eldermeier (Karlsrhue, Germany). This work was supported by the Fundação para a Ciência e Tecnologia (POCTI/AGR/40283/2001 project) and GRICES (Proc. 
423 ICCTI/CSIC). B. Silva is indebted to Fundação para a Ciência e Tecnologia for a Ph.D. Grant (SFRH/ $\mathrm{BD} / 13488 / 2003)$.

\section{References}

Agostinis, P., Vantieghem, A., Merlevede, W., \& de Witte, P. A. (2002). Hypericin in cancer treatment: More light on the way. The International Journal of Biochemistry and Cell Biology, 34, 221-241.

Areias, F. M., Rego, A. C., Oliveira, C. R., \& Seabra, R. M. (2001). Antioxidant effect of flavonoids after ascorbate $/ \mathrm{Fe}^{2+}$-induced oxidative stress in cultured retinal cells. Biochemical Pharmacology, 62, 111-118.

Behl, C., \& Mosmann, B. (2002). Antioxidant neuroprotection in Alzheimer's disease as preventive and therapeutic approach. Free Radical Biology and Medicine, 33, 182-191.

Ben-Yoseph, O., Boxer, P. A., \& Ross, B. D. (1996). Assessment of the role of the glutathione and pentose phosphate pathways in the protection of primary cerebrocortical cultures from oxidative stress. Journal of Neurochemistry, 66, 2329-2337.

Butterweck, V. (2003). Mechanism of action of St. John's wort in depression: What is known? CNS Drugs, 17, 539-562.

Conforti, F., Statti, G. A., Tundis, R., Menichini, F., \& Houghton, P. (2002). Antioxidant activity of methanolic extract of Hypericum triquetrifolium Turra aerial part. Fitoterapia, 73, 479-483.

Cos, P., Calomme, M., Sindambiwe, J. B., de Bruyne, T., Cimanga, K., Pieters, L., Vlietinck, A. J., \& Vanden Berghe, D. (2001). Cytotoxicity and lipid peroxidation-inhibiting activity of flavonoids. Planta Medica, 67, 515-519.

Cuyckens, F., Rozenberg, R., Hoffmann, E., \& Claeys, M. (2001). Structure characterization of flavonoid O-diglycosides by positive and negative nano-electrospray ionization ion trap mass spectrometry. Journal of Mass Spectrometry, 36, 1203-1210.

Di Carlo, G., Borrelli, F., Ernst, E., \& Izzo, A. A. (2001). St John's wort: Prozac from the plant kingdom. Trends in Pharmacological Sciences, 22, 292-297.

Dias, A. C. P., Seabra, R. M., Andrade, P. B., \& Ferreira, M. F. (1999). The development and evaluation of a HPLC-DAD method for the analysis of the phenolic fractions from in vivo and in vitro biomass of Hypericum species. Journal of Liquid Chromatography, 22, 215-227.

Dias, A. C. P., \& Ferreira, M. F. (2003). Production of phenolics by in vitro cultures of Hypericum perforatum : A case study. In A. P. Rauter, M. E. Araújo, F. B. Palma, J. Justino, \& S. P. Santos (Eds.), Natural products in the new millenium: Prospects and industrial applications (pp. 367-374). Kluwer Academic Publishers.

Erdelmeier, C. A. J., Koch, E., \& Hoerr, R. (2000). Hypericum perforatum - St. John's wort chemical, pharmacological and clinical aspects. In Atta-ur-Rahman (Ed.), Studies in natural products chemistry - bioactive natural products (Part C) (Vol. 22, pp. 643-716). New York: Elsevier Direct.

Foy, C. J., Passmore, A. P., Vahidassr, M. D., Young, I. S., \& Lawson, J. T. (1999). Plasma chain-breaking antioxidants in Alzheimer's disease, vascular dementia, and Parkinson's disease. QJM Journal of the Association of Physicians, 92, 39-45.

Fridovich, I. (1997). Superoxide anion radical $\left(\mathrm{O}_{2}^{-\cdot}\right)$, superoxide dismutases, and related matters. Journal of Biological Chemistry, 272, 18,515-18,517.

Fukumoto, L. R., \& Mazza, G. (2000). Assessing antioxidant and prooxidant activities of phenolic compounds. Journal of Agricultural and Food Chemistry, 48, 3597-3604.

Fuzzati, N., Gabetta, B., Strepponi, I., \& Villa, F. (2001). Highperformance liquid chromatography-electrospray ionization mass spectrometry and multiple mass spectrometry studies of hyperforin degradation products. Journal of Chromatography A, 926, 187-198.

Gaedcke, F. (2003). St John's wort herb extracts: Manufacturing, standardisation and characterization. In E. Ernst (Ed.), Hypericum, medicinal and aromatic plants - industrial profiles (Vol. 31, pp. 49 64). London: Taylor and Francis Group.

Gardner, P. T., White, T. A. C., McPhail, D. B., \& Duthie, G. G. (2000). The relative contributions of vitamin C, carotenoids and phenolics to the antioxidant potential of fruit juices. Food Chemistry, 68, 471-474.

Gutmann, H., Bruggisser, R., Schaffner, W., Bogman, K., Botomino, A., \& Drewe, J. (2002). Transport of amentoflavone across the blood-brain barrier in vitro. Planta Medica, 68, 804-807.

Halliwell, B., Aeschbach, R., Löliger, J., \& Aruoma, O. I. (1995). The characterization of antioxidants. Food and Chemical Toxicology, $33,601-617$

Halliwell, B. (1996). Oxidative stress, nutrition and health. Experimental strategies for optimization of nutritional antioxidant intake in humans. Free Radical Research, 25, 57-74.

Halliwell, B. (1999). Establishing the significance and optimal intake of dietary antioxidants: The biomarker concept. Nutrition Reviews, 57, 104-113.

Hunt, E. J., Lester, C. E., Lester, E. A., \& Tackett, R. L. (2001). Effect of St. John's wort on free radical production. Life Sciences, 69, 181-190.

Hyun, D. H., Lee, M. H., Halwell, B., \& Jenner, P. (2002). Proteasomal dysfunction induced by 4-hydroxy-2,3-trans-nonenal, an end-product of lipid peroxidation: A mechanism contributing to neurodegeneration? Journal of Neurochemistry, 83, 360-370.

Jürgenliemk, G., \& Nahrstedt, A. (2002). Phenolic compounds from Hypericum perforatum. Planta Medica, 68, 88-91.

Kasper, S. (2001). Hypericum perforatum - a review of clinical studies. Pharmacopsychiatry, 34(Suppl 1), S51-S55.

Ko, F. N., Cheng, Z. J., Lin, C. N., \& Teng, C. M. (1998). Scavenger and antioxidant properties of prenylflavones isolated from Artocarpus heterophyllus. Free Radical Biology and Medicine, 25, 160168.

Kurth, H., \& Spreemann, R. (1998). Phytochemical characterization of various St. John's wort extracts. Advances in Therapy, 15, 117-128.

LaFrance, W. C., Jr., Lauterbach, E. C., Coffey, C. E., Salloway, S. P., Kaufer, D. I., Reeve, A., Royall, D. R., Aylward, E., Rummans, T. A., \& Lovell, M. R. (2000). The use of herbal alternative medicines in neuropsychiatry. A report of the ANPA Committee on Research. The Journal of Neuropsychiatry and Clinical Neurosciences, 12, 177-192.

Lebeau, J., Furman, C., Bernier, J.-L., Duriez, P., Teissier, E., \& Cotelle, N. (2000). Antioxidant properties of di-tert -butylhydroxylated flavonoids. Free Radical Biology and Medicine, 29, 900912.

Li, C., \& Xie, B. (2000). Evaluation of the antioxidant and pro-oxidant effects of tea catechin oxypolymers. Journal of Agricultural and Food Chemistry, 48, 6362-6366.

Markesbery, W. R. (1997). Oxidative stress hypothesis in Alzheimer's disease. Free Radical Biology and Medicine, 23, 134-147.

Miranda, C. L., Stevens, J. F., Ivanov, V., McCall, M., Frei, B., Deinzer, M. L., \& Buhler, D. R. (2000). Antioxidant and prooxidant actions of prenylated and nonprenylated chalcones and flavanones in vitro. Journal of Agricultural and Food Chemistry, 48, 3876-3884.

Pereira, C. F., Moreira, P., Seica, R., Santos, M. S., \& Oliveira, C. R. (2000). Susceptibility to $\beta$-amyloid-induced toxicity is decreased in goto-kakizaki diabetic rats: Involvement of oxidative stress. Experimental Neurology, 161(1), 383-391.

Prince, A. M., Pascual, D., Meruelo, D., Liebes, L., Mazur, Y., Dubovi, E., Mandel, M., Rodriguez, R. J., Miranda, C. L., Stevens, J. F., Deinzer, M. L., \& Buhler, D. R. (2001). Influence of prenylated and non-prenylated flavonoids on liver microsomal lipid 
peroxidation and oxidative injury in rat hepatocytes. Food Chemical Toxicology, 39, 437-445.

Rice-Evans, C. A., Miller, N. J., \& Paganga, G. (1997). Antioxidant properties of phenolic compounds. Trends in Plant Science, 2, 152 159.

Schroeter, H., Williams, R. J., Matin, R., Iversen, L., \& Rice-Evans, C. A. (2000). Phenolic antioxidants attenuate neuronal cell death following uptake of oxidized low-density lipoprotein. Free Radical Biology and Medicine, 29, 1222-1233.

Silva, A. P., Carvalho, A. P., Carvalho, C. M., \& Malva, J. O. (2001). Modulation of intracellular calcium changes and glutamate release by neuropeptide $\mathrm{Y} 1$ and $\mathrm{Y} 2$ receptors in the rat hippocampus: Differential effects in CA1, CA3 and dentate gyrus. Journal of Neurochemistry, 79, 286-296.

Silva, B. C., \& Dias, A. C. P. (2002). Evaluation of the free radical scavenger activity of Hypericum perforatum alcoholic extracts. Revista de Fitoterapia, 2(Suppl 1), 133.

Tolonen, A., Uusitalo, J., Hohtola, A., \& Jalonen, J. (2002). Determination of naphthodianthrones and phloroglucinols from Hypericum perforatum extracts by liquid chromatography/tandem mass spectrometry. Rapid Communications in Mass Spectrometry, 16, 396-402.

Valentao, P., Fernandes, E., Carvalho, F., Andrade, P. B., Seabra, R. M., \& de Lourdes Bastos, M. (2002). Antioxidant activity of Hypericum androsaemum infusion: Scavenging activity against superoxide radical, hydroxyl radical and hypochlorous acid. Biological and Pharmaceutical Bulletin, 25, 1320-1323.

Wills, R. B. H., Bone, K., \& Morgan, M. (2000). Herbal products: Active constituents, modes of action and quality control. Nutritional Research Reviews, 13, 47-77.

Youdim, K. A., Spencer, J. P., Schroeter, H., \& Rice-Evans, C. (2002). Dietary flavonoids as potential neuroprotectants. Biological Chemistry, 383, 503-519.

Youdim, K. A., Dobbie, M. S., Kuhnle, G., Proteggente, A. R., Abbott, N. J., \& Rice-Evans, C. (2003). Interaction between flavonoids and the blood-brain barrier: In vitro studies. Journal of Neurochemistry, 85, 180-192.

Zheng, W., \& Wang, S. Y. (2001). Antioxidant activity and phenolic compounds in selected herbs. Journal of Agricultural and Food Chemistry, 49, 5165-5170. 